\title{
Power Flow Tracing for Transmission Open Access
}

\author{
Ping Wei (St. M. IEEE) Bin Yuan Yixin Ni (S. M. IEEE) Felix F. Wu (Fellow IEEE) \\ Department of Electrical and Electronic Engineering \\ The University of Hong Kong \\ Hong Kong
}

\begin{abstract}
In this paper, graph theory is used to analyze real power transfer between individual generators and loads, which is important to transmission open access. Related lemmas are proved first to present necessary conditions required by the method. Based on an ac load flow solution a novel method is suggested which can determine downstream and upstream power flow tracing paths and calculate the contribution factors of generations and loads to the line flows efficiently in the system without circulating power. When the existence of circulating power in the system is detected, by revealing the reasons which cause circulating power, the optimal power flow (OPF) approach is suggested to eliminate it, and a new feasible ac load flow solution will be obtained for tracing. The computer tests yield satisfactory results. The suggested method is suitable for both active and reactive power flow tracings of real power systems.
\end{abstract}

Keywords: graph theory, transmission open access, power flow tracing, optimal power flow.

\section{INTRODUCTION}

Power system deregulation and transmission open access make it more and more important to calculate the contributions of individual generators and loads to line flows and the real power transfers between individual generators and loads. The results can help to allocate the total cost of transmission among all the users in an equitable way.

Methods based on de load flow and sensitivity analysis can't consider accurately the reactive power transfer allocation and system non-linearity. In $[1,2]$ a novel method is suggested to solve the problem however a matrix inverse calculation is required which is time consuming especially for a large-scale power system. In [3] another approach is presented which

Paper accepted for presentation at the International Conference on Electric Utility Deregulation and Restructuring and Power Technologies 2000, City University, London, 4-7 April 2000.

0-7803-5902-X/00/\$10.00 @2000 IEEE. introduces some new concepts such as domain, common, link and state graph and is suitable for large-scale power system applications. However there are no clear declaration and proof of the conditions required for the method.

In this paper graph theory [4] is suggested to solve the problem. Graph theory is quite mature and especially suitable to tackle such network topology related issues. In our case a directed graph is used. The vertices of the graph are system buses and the edges of the graph are lines and transformers. The direction of each edge is the direction of power flow inside. The directed graph of active power flow may be different from that of reactive power flow in edge directions. Based on an ac load flow solution, the suggested method can consider system non-linearity accurately and is very efficient and suitable for both active and reactive power transfer allocations in real power systems.

The paper is organized as follows. In section 2, two lemmas are proved firstly which presents the necessary conditions to guarantee the feasibility of the suggested method in power flow tracing in a system without circulating power. Then another two lemmas are proved to reveal the reasons for circulating power. Using active power as an example, graph theory is applied to determine the tracing sequence and to detect the existence of circulating power. In section 3 an optimal power flow (OPF) [5] approach is suggested to eliminate circulating power. The sequential quadratic programming [6] is used for OPF solution. In section 4 through upstream and downstream tracings, contribution factors of individual generators and loads to line flows are calculated respectively. The power transfers between generators and loads are determined as well. Section 5 presents the test results from a 6-bus system. Conclusions are made in section 6 .

\section{FUNDAMENTALS OF LOAD FLOW TRACING}

In order to simplify the problem, some assumptions are made at first:

- An ac load flow solution is available from on-line state estimation or off-line system analysis.

- Real power and reactive power required by transmission line resistance, reactance and charging capacitance have been moved to the line terminal buses and modeled as 'equivalent loads' according to ac load flow solution. Therefore the line active and reactive power flows keep constant along the line, each edge has a definite direction and the network is 'lossless'. 
- A generator has the priority to provide power to the load on the same bus, and the remaining power will enter the network to supply other loads in the network to avoid unnecessary losses. Therefore the buses can be classified as generator, load and network buses based on their net injections to the system.

- The flows of electricity obey the proportional-sharing rule [1-3].

Based on the assumptions made above, we can prove the following two lemmas, which guarantee to start and continue a tracing:

Lemma 1: A lossless, finite-nodes power system without circulating flow has at least one pure source, i.e. a generator bus with all incident lines carrying outflows.

Lemma 2: A lossless, finite-nodes power system without circulating flow has at least one pure sink, i.e. a load bus with all incident lines carrying inflows.

Proof of Lemma 1: We start from any bus with an incident line carrying inflows. Along this line we go upstream and come to a next bus (here we assume there is no self-loop). If there is no incident line carrying inflows to the next bus, then it is an existing pure sink already. If there is at least one incident line carrying inflow to the next bus, then we can continue to go upstream along the line to another bus. This upstream tracing can continue if there is no pure source confronted. If the upstream bus is always a new bus, this will lead to the conclusion that the system has infinite buses. If the upstream bus is an old bus, that is a bus appeared in the previous tracing path, then a circulating flow is existing in the system which conflicts with the assumption. Therefore a pure source does exist to end the upstream tracing.

Proof of Lemma 2: We start from any bus with an incident line carrying outflows. Along this line we go downstream and come to a next bus (still we assume there is no self-loop). If there is no incident line carrying outflows from the next bus, then it is an existing pure sink already. If there is at least one incident line carrying outflow from the next bus, then we can continue to go downstream along the line to another bus. This downstream tracing can continue if there is no pure sink confronted. If the downstream bus is always a new bus, this will lead to the conclusion that the system has infinite buses. If the downstream bus is an old bus, that is a bus appeared in the previous tracing path, then a circulating flow is existing in the system which conflicts with the assumption. Therefore a pure sink does exist to end the downstream tracing.

In well-operated power systems, there should not be circulating power because the circulating power will cause additional losses and have negative impacts on power transfer capability and voltage regulation. However sometimes circulating power does exist due to the mal-operation control, the system complexity and poor controllability. Now we prove another two lemmas to show the reasons for the circulating power.

Lemma 3: For a lossless transmission network, the only reason for active power circulation is the existence of phase shifting device(s).

Lemma 4: For a lossless transmission network, the only reason for reactive power circulation is the existence of voltage regulator(s).

Proof of Lemma 3: For a lossless transmission line, active power flow over the line is

$$
P_{i j}=\frac{V_{i} V_{j}}{x_{i j}} \sin \left(\theta_{i}-\theta_{j}\right)
$$

where $V_{i}$ and $V_{j}$ are the line terminal voltage magnitudes, $x_{i j}$ is the line reactance and $\theta_{i}$ and $\theta_{j}$ are phase angles of line terminal voltages. If $P_{i j}>0$, then $\sin \left(\theta_{i}-\theta_{j}\right)$ must be positive. In power system for stable operation the line terminal phase angle difference $\left(\theta_{i}-\theta_{j}\right)$ should be within $[-90,90]$ degrees. So from $\sin \left(\theta_{i}-\theta_{j}\right)>0$ we can conclude that $\left(\theta_{i}-\theta_{j}\right)>0$ or $\theta_{i}>\theta_{j}$ which means that in a lossless transmission system, active power always flows from the point with larger phase angle to the point with smaller phase angle. This is also true for a conventional transformer with only voltage magnitude regulation capability. Therefore if a lossless transmission system has circulating active power, then in the circulating active power loop there must be at least one branch where the active power flows from a bus with smaller phase angle to a bus with langer phase angle. This branch must not be a transmission line or a conventional transformer with only voltage magnitude regulation capability. It must be a device with phase-shifting capability and active power can flow through it from a terminal with smaller phase angle to a terminal with larger phase angle. It is clear that if there is no phase-shifting devices in a lossless transmission system, there will be no active power circulation.

The possible devices with phase-shifting capability are phase shifters (solid-state or conventional), FACTS devices such as UPFC with series element working as a phase shifter and $Y / \Delta$-connection transformers etc.

Proof of Lemma 4: For a lossless transmission system, if the $\pi$ equivalence circuit is used for the transmission lines and the charging capacitance has been merged into the reactive loads on line terminal buses, then the reactive power reaching the receiving end of a line can be expressed as:

$$
\begin{aligned}
Q_{j} & =\operatorname{Im}\left(\dot{V}_{j}\left[\left(\dot{V}_{i}-\dot{V}_{j}\right) / j x_{i j}\right]^{*}\right\} \\
& =\left(V_{i} \cos \theta_{i j}-V_{j}\right) V_{j} / x_{i j}
\end{aligned}
$$

It is clear from eq. (2) that if $Q_{j}>0$ then $V_{i}$ must be greater then $V_{j}$ since $\cos \theta_{i j}$ is within the range of $[0,1]$ in a normal power system. This means that if some reactive power reaches a line terminal then it must flow from a terminal with higher voltage to a terminal with lower voltage for a lossless transmission system. Therefore if a lossless transmission system has circulating reactive power, then in the circulating reactive power loop there must be at least one branch where the reactive power flows from a bus with lower voltage to a bus with higher voltage. This branch must not be a transmission line. It must be a device with voltage regulation capability and 
reactive power can flow through it from a terminal with lower voltage to a terminal with higher voltage. It is clear that if there is no voltage regulation devices in a lossless transmission system, there will be no reactive power circulation.

The possible devices with voltage regulation capability in power systems are conventional transformers/voltage regulators and the FACTS devices such as UPFC with its series element working as a transformer or voltage regulator etc. It is clear that any shunt types of FACTS devices will not cause circulating reactive power.

Since the line $X / R$ ratio is very high for high voltage transmission systems, we can consider the conclusions of the last two lemmas are still thue, i. e. the phase-shifter and the voltage regulator cause the active and reactive power circulating respectively in a real transmission system.

For a transmission system, we can build up its bus-line incident matrix (BLIM) according to the load flow solution and line active power flow direction. Then split the BLIM into two matrices. One is called bus-outflow-line incident matrix (BOLIM) which is composed of all elements in BLIM with the value of 1 , and the other is called bus-inflow-line incident matrix (BILIM) which is composed of all elements in BLIM with the value of $(-1)$ and these elements are then changed into 1. For a 6-bus sample system shown in figure 1(a), the corresponding BLIM, BOLIM and BILIM are formed in figure 1(b). It is clear that a row with full zero elements in BOLIM corresponds to a pure sink and a row with full zero elements in BILIM corresponds to a pure source.

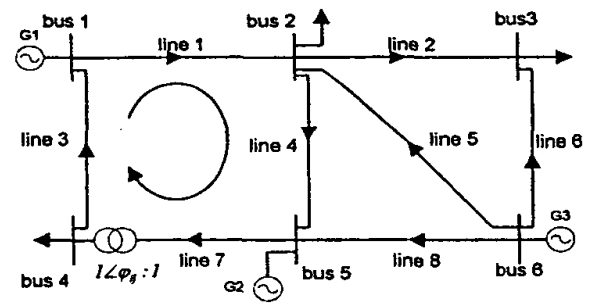

$(\rightarrow$ : active power flow direction )

(a)

\begin{tabular}{|c|c|c|c|c|c|c|c|c|c|}
\hline & Lir & e 1 & 2 & 3 & 4 & 5 & 6 & 7 & 8 \\
\hline & bus 1 & 1 & 0 & -1 & 0 & 0 & 0 & 0 & 0 \\
\hline & 2 & -1 & 1 & 0 & 1 & -1 & 0 & 0 & 0 \\
\hline & 3 & 0 & -1 & 0 & 0 & 0 & -1 & 0 & 0 \\
\hline BLIM & 4 & 0 & 0 & 1 & 0 & 0 & 0 & -1 & 0 \\
\hline & 5 & 0 & 0 & 0 & -1 & 0 & 0 & 1 & -1 \\
\hline & 6 & 0 & 0 & 0 & 0 & 1 & 1 & 0 & 1 \\
\hline
\end{tabular}

BOLIM $=\left[\begin{array}{llllllll}1 & 0 & 0 & 0 & 0 & 0 & 0 & 0 \\ 0 & 1 & 0 & 1 & 0 & 0 & 0 & 0 \\ 0 & 0 & 0 & 0 & 0 & 0 & 0 & 0 \\ 0 & 0 & 1 & 0 & 0 & 0 & 0 & 0 \\ 0 & 0 & 0 & 0 & 0 & 0 & 1 & 0 \\ 0 & 0 & 0 & 0 & 1 & 1 & 0 & 1\end{array}\right]$ BILIM $\left[\begin{array}{llllllll}0 & 0 & 1 & 0 & 0 & 0 & 0 & 0 \\ 1 & 0 & 0 & 0 & 1 & 0 & 0 & 0 \\ 0 & 1 & 0 & 0 & 0 & 1 & 0 & 0 \\ 0 & 0 & 0 & 0 & 0 & 0 & 1 & 0 \\ 0 & 0 & 0 & 1 & 0 & 0 & 0 & 1 \\ 0 & 0 & 0 & 0 & 0 & 0 & 0 & 0\end{array}\right]$

(b)

Figure 1 A 6-bus system and its BLIM, BOLIM and BILIM
Downstream Tracing Sequence: We start from a pure source (bus 6 for our case, see BILIM). Outflow lines of bus 6 will bring generator power forward to their downstream buses. Delete the source bus and its incident lines (This is done by deleting row 6 and corresponding columns of its incident lines, i.e. columns 5,6 and 8 , which have the element 1 in row 6 of BOLIM). We have a new graph that is a sub-graph of original one. Then try to find the new pure source bus in the sub-graph together with its incident (outflow) lines and repeat the deleting process described above again. If there is no circulating power in the system, the downstream tracing can be continued through this process till all the lines are deleted with only pure sink(s) remaining. However for our case, the downstream tracing can only delete bus 6 with lines $5,6,8$ and then stop since there is circulating power existing.

Upstream Tracing Sequence: Similar process can be performed by starting from a pure sink. If there is no circulating power in the system, the upstream tracing can be continued till all the lines are deleted with only pure source(s) remaining. However the upstream tracing of our test system can only delete bus 3 with lines 2, 6 and then cannot find pure sink existing because of circulating power.

Detection of Circulating Power: After downstream and upstream tracing are conducted, the remaining part will be the area with circulating power since each bus has both inflow and outflow lines. If a transmission system has circulating power, through this process the area(s) with circulating power can be detected.

\section{ELIMINATION OF CIRCULATING POWER}

From lemma 3 we know that the circulating active power is caused by phase shifting devices. We also know the circulating power will cause additional losses. Therefore we can use optimal power flow (OPF) approach [5] to eliminate the circulating power by minimizing the transmission system losses, and the phase shifter angle will be the control variable in OPF. In order to save CPU time OPF is conducted only in the rest of the system after downstream and upstream tracing. The deleted part can be simplified through static equivalence technique or just modeled as equivalent power injections to the remaining part. After OPF, the new phase shifting angle will be used for the original system and a global system load flow will be calculated for later use. If there is no circulating power existing under the new phase shifting angle, the task of circulating power elimination is completed.

Sometimes iterations are required since after the OPF solution and the new global system load flow calculation, the injection power from the 'deleted' part to the rest might change significantly or some operation limits are violated. Therefore another round of OPF solution and the load flow calculation might be required.

The OPF problem for elimination of circulating power can be formulated as follows. The objective function takes the 
form:

$$
\min _{\Phi_{i}} f=\sum_{\text {line } i j} \frac{V_{i}^{2}+V_{j}^{2}-2 V_{i} V_{j} \cos \left(\theta_{i}-\theta_{j}-\varphi_{i j}\right)}{r_{i j}^{2}+x_{i j}^{2}} \cdot r_{i j}
$$

where $V_{i,} \theta_{i}$ : voltage magnitude and phase angle of bus $i ; r_{i j}, x_{i j}$ : resistance and reactance of line $i-j ; \varphi_{i j}$ : adjustable angle of phase shifter installed on line $i-j$.

The equality constraints are power flow equations:

$$
\begin{aligned}
& P_{g i}-P_{d i}-\sum_{j \in N} V_{i} V_{j} Y_{i j} \cos \left(\theta_{i j}-\delta_{i j}-\varphi_{i j}\right)=0 \\
& Q_{g i}-Q_{d i}-\sum_{j \in N} V_{i} V_{j} Y_{i j} \sin \left(\theta_{i j}-\delta_{i j}-\varphi_{i j}\right)=0
\end{aligned}
$$

where $P_{g i}, Q_{g i}, P_{d i}, Q_{d i}$ : active and reactive generation and demand at bus $i$; $Y_{i j}$, $\delta_{i j}$ : the element of bus admittance matrix and the corresponding phase angle.

The inequality constraints are as follows:

- Phase shifter control angle limits:

$$
\varphi_{i j, \min } \leq \varphi_{i j} \leq \varphi_{i j, \text { max }}
$$

for a line without phase shifter, $\varphi_{i j}=0$.

- Transmission line limits:

$$
\left|P_{i j}\right| \leq P_{i j, \max }
$$

where

$$
P_{i j}=\operatorname{Re}\left\{\dot{V}_{i} e^{-j \varphi_{i j}}\left[\left(\dot{V}_{i} e^{-j \varphi_{i j}}-\dot{V}_{j}\right) /\left(r_{i j}+j x_{i j}\right)\right]^{*}\right\}
$$

- Bus voltage limits:

$$
V_{i, \min } \leq V_{i} \leq V_{i, \max }
$$

The sequential quadratic method [6] is used to solve the OPF problem.

\section{CONTRIBUTION AND EXTRACTION FACTORS}

For a system without circulating flow, the power flow tracing can be performed similar to [7] which is outlined below.

\subsection{Downstream Tracing}

The downstream tracing is used for calculating the contribution factors of individual generators to line flows and loads. The tracing sequence is determined by the method suggested in Section 2, and the ac load flow solution without circulating power is provided by the method suggested in Section 3.

To solve the problem, we need to build up two matrices. One is extraction factor matrix of lines $\left(A_{l}\right)$ and loads $\left(A_{L}\right)$ from bus total passing power of their upstream buses. The other is contribution factor matrix $(B)$ of generators to bus total passing power $(P)$. The product of these two matrices constitutes the contribution factors of generators to line flows $\left(K_{I G}\right)$ and loads $\left(K_{L G}\right)$. That is:

$$
\begin{aligned}
& P_{l}=A_{l} P=A_{l} B P_{G}=K_{l G} P_{G} \\
& P_{L}=A_{L} P=A_{L} B P_{G}=K_{L G} P_{G}
\end{aligned}
$$

where $P_{l}, P_{L}, P$ and $P_{G}$ are the vectors of line power, load power, total bus passing power and generator power in downstream sequence respectively.

The non-zero elements in $A_{l}$ is calculated as follows:

$$
\left(A_{l}\right)_{\text {line } j, b u s i}=\frac{\text { line } j^{\prime} s \text { power flow }}{\text { bus } i^{\prime} \text { s total pas sing power } P_{i}}
$$

where bus $i$ is the upstream terminal bus of line $j$.

$A_{L}$ is a diagonal matrix with indication of net load buses and can be calculated as;

$$
A L_{i i}= \begin{cases}0 & i \notin \text { net load buses } \\ \frac{\text { net load power on bus } i}{P_{i}} & i \in \text { net load buses }\end{cases}
$$

The elements in matrix $B$ is calculated row by row as:

$B_{b u s-i, b u s-k}=\left\{\begin{array}{cc}1 & (k=i, k \in \text { net gen.buses }) \\ 0 & (k=i, k \notin \text { net gen. buses }) \\ 0 \quad(k<i, k \notin \text { net gen. buses }) \\ \sum_{I_{j} \in i}\left(A_{l_{j}-m} \cdot B_{m-k}\right)(k<i, k \in \text { net gen.buses })\end{array}\right.$

where ' $k<i$ ' means $k$ is an upstream bus of bus $i$, and ' $k>i$ ' means $k$ is a downstream bus of bus $i$. The term ' $l_{j} \varepsilon i$ ' means line $j$ is an inflow line of bus $i . A_{l_{j}-m}$ is the unique non-zero element corresponding to line $j$ in matrix $A_{l}$ with bus $m$ as its upstream terminal. $B_{m-k}$ is the element in matrix $B$ already calculated which represents the contribution of generator $k$ to the total injection power of bus $m$. The product $\left(A_{l_{j}-m} \cdot B_{m-k}\right)$ represents the contribution of generator $k$ to the total injection power of bus $i$ through line $j$ (from bus $m$ to bus $i$ ).

However we are not able to get extraction factors of loads from generators through downstream tracing since the state variable in the downstream tracing is the generator power. These factors can only be obtained from the upstream tracing which takes the load power as the state variable.

\subsection{Upstream Tracing}

The upstream tracing is used for calculating the extraction factors of loads from line flows and generators. The tracing sequence is determined by the method suggested in Section 2 and beginning from a pure sink bus. Similarly we build up two matrices. One is contribution factor matrix of lines $\left(C_{l}\right)$ and generators $\left(C_{G}\right)$ to bus total passing power. The other is extraction factor matrix $(D)$ of loads from bus total passing power. The product of these two matrices constitutes the extraction factors of loads from line flows $\left(K_{l L}\right)$ and generators $\left(K_{G L}\right)$. Then we get: 


$$
\begin{aligned}
& P_{l}=C_{l} P=C_{l} D P_{L}=K_{l L} P_{L} \\
& P_{G}=C_{G} P=C_{G} D P_{L}=K_{G L} P_{L}
\end{aligned}
$$

where $P_{l}, P_{L} P$ and $P_{G}$ have the same physical meaning as in downstream tracing, but with their elements in the upstream sequences.

The non-zero element in $C_{l}$ is determined as follows:

$$
\left(C_{l}\right)_{\text {line } j, \text { bus } i}=\frac{\text { line } j^{\prime} s \text { power flow }}{\text { bus } i^{\prime} \text { s total passing power } P_{i}}
$$

where bus $i$ is the downstream terminal bus of line $j$.

Matrix $C_{G}$ is a diagonal matrix with indication of net generator buses. It can be calculated as:

$$
C_{G_{i i}}=\left\{\begin{array}{lc}
0 & i \notin n \text { net gen. buses } \\
\frac{\text { net gen. power on bus } i}{P_{i}} & i \in \text { net gen.buses }
\end{array}\right.
$$

The elements in matrix $\mathrm{D}$ is formed row by row as:

$$
D_{\text {bus }-i, \text { bus }-k}=\left\{\begin{array}{cc}
1 & (k=i, k \in \text { net load. buses }) \\
0 & (k=i, k \notin \text { netload. buses }) \\
0 & (k>i) \\
0 & (k<i, k \notin \text { net load buses }) \\
\sum_{I_{j}{ }^{i}}\left(C_{l_{j}-m} \cdot D_{m-k}\right) & (k<i, k \in \text { netload buses })
\end{array}\right.
$$

where ' $k<i$ ' means bus $k$ is a downstream bus of bus $i$ and ' $k>i$ ' means bus $\mathrm{k}$ is an upstream bus of bus $i$. The term ' $l_{j} \varepsilon i$ ' means that line $j$ is an outflow line of bus $i . c_{l_{j}-m}$ is the unique non-zero element corresponding to line $j$ (from bus $i$ to bus $m$ ) in matrix $C_{l} . D_{m-k}$ is the element in matrix $D$ already calculated which represents the extraction factor of load $k$ from the total passing power of bus $m$. The product $\left(c_{l_{j}-m} \cdot D_{m-k}\right)$ represents the extraction of load $k$ from the total outflow power of bus $i$ through line $j$ (from bus $i$ to bus $m$ ).

\section{COMPUTER TEST RESULTS}

The suggested algorithm is tested on a 6-bus sample system shown in Figure 1. The system parameters are listed in Table A of the appendix. There is only one phase shifter installed on line 7 , which causes circulating power. The original system load flow solution is listed in Table B of the appendix. The total system loss is $0.0407 \mathrm{pu}$ from ac load flow.

Through upstream and downstream tracing in Section 2, the sub-system containing circulating power can be detected and isolated from original system. It is composed of buses $1,2,4,5$ and lines with circulating power.

Take the power flow on lines 2,5 and 8 as equivalent injection power on bus 2 and bus 5 and optimize the control angle of phase shifter to minimize the total loss of the subsystem by using the method suggested in Section 3 . The phase shifter angle changes from the original 12 degrees to -1.708 degree. Taking that value back to the original system, keeping the generator and load power as constant except swing bus and running a global system load flow, the new load flow solution is shown in Table B of the APPENDIX as well. There is no circulating power or constraint violation. The system loss is reduced from 0.0407 p.u. to 0.0138 p.u. The difference should be taken care of by the system operator for poor control of the phase shifter angle.

Using the new load flow solution without circulating power, power flow tracing can be conducted through the method introduced in Section 4. The active power transferred from each generator to each load is listed in Table 1.

Table 1. Active power flow tracing (MW)

\begin{tabular}{|c|c|c|c|c|}
\hline $\begin{array}{c}\text { Bus } \\
\text { No. }\end{array}$ & Load & $\begin{array}{c}\text { Supplied } \\
\text { by G1 }\end{array}$ & $\begin{array}{c}\text { Supplied } \\
\text { by G2 }\end{array}$ & $\begin{array}{c}\text { Supplied } \\
\text { by G3 }\end{array}$ \\
\hline 1 & 0 & 0.00 & 0.00 & 0.00 \\
\hline 2 & 100 & 33.59 & 50.88 & 15.53 \\
\hline 3 & 80 & 4.90 & 11.14 & 63.97 \\
\hline 4 & 60 & 42.42 & 17.57 & 0.00 \\
\hline 5 & 0 & 0.00 & 0.00 & 0.00 \\
\hline 6 & 0 & 0.00 & 0.00 & 0.00 \\
\hline Loss & $(1.38)$ & 0.47 & 0.41 & 0.50 \\
\hline Total & 241.38 & 81.38 & 80 & 80 \\
\hline
\end{tabular}

The suggested method is also tested on the IEEE14-bus and the IEEE30-bus systems. Satisfactory results are obtained as well. The results will not be presented in the paper.

\section{CONCLUSION}

In this paper graph theory is applied to calculate the contribution factors of individual generators to line flows and loads and the extraction factors of individual loads from line flows and generators in a system without circulating power. Also by using graph theory, the existing of circulating power can be detected easily. Then OPF method is suggested to eliminate the circulating power and provide a new feasible ac load flow solution for power flow tracing. The computer tests on several power systems yield satisfactory results. The suggested method is very efficient and suitable for use in real power systems.

\section{REFERENCES}

[1] J. Bialek, Tracing the flow of electricity, IEE Proceedings-Generation Transmission \& Distribution, Vol. 143, No.4, July 1996.

[2] J. Bialek, Topological generation and load distribution 
factors for supplementary charge allocation in transmission open access, IEEE Trans. on PWRS, Vol. 12, No.3, Aug. 1997.

[3] . D. Kirschen, et al., Contribution of individual generators to loads and flows, IEEE Trans. On PWRS, Vol. 12, No.1, Feb. 1997.

[4] Y. Alavi, et al., Graph theory, combinatorics, algorithms and applications, Society for Industrial and Applied Mathematics, 1991.

[5] J. A. Momoh, M. E. El-Hawary and R. Adapa, $A$ review of selected optimal power flow literature to 1993, Part II: Newton, linear programming and interior point methods, IEEE Transactions on PWRS, Vol. 14, No. 1, Feb. 1999.

[6] S. S. Rao, Engineering Optimization: Theory and Practice, John Wiley \& Sons, 3rd edition, 1996.

[7] Felix F. Wu, Y. Ni, P. Wei, Power transfer allocation for Dr. Eng. degrees all in electrical engineering, Tsinghua open access using graph theory- fundamentals and University, China. She was former professor and director of applications in systems without loop-flow, to be appeared in National Power System Lab, Tsinghua University and now IEEE Trans. on Power Systems.

\section{APPENDIX}

Table A. Line Parameters of the 6-Bus System (pu)

\begin{tabular}{|c|c|c|c|c|c|c|}
\hline $\begin{array}{c}\text { Line } \\
\text { No. }\end{array}$ & $\begin{array}{c}\text { From-To } \\
\text { Bus No. }\end{array}$ & $\mathrm{R}$ & $\mathrm{X}$ & $\mathrm{B}_{\mathrm{c}}$ & $\begin{array}{c}\text { Phase } \\
\text { Shifter } \\
\text { Angle ( }\end{array}$ & $\begin{array}{c}\text { Max. } \\
\text { Power }\end{array}$ \\
\hline 1 & $1-2$ & 0.017 & 0.092 & 0.158 & 0 & 1.5 \\
\hline 2 & $2-3$ & 0.039 & 0.170 & 0.358 & 0 & 1.5 \\
\hline 3 & $1-4$ & 0.012 & 0.101 & 0.209 & 0 & 1.5 \\
\hline 4 & $2-5$ & 0.009 & 0.072 & 0.149 & 0 & 1.5 \\
\hline 5 & $2-6$ & 0.032 & 0.161 & 0.306 & 0 & 1.5 \\
\hline 6 & $3-6$ & 0.010 & 0.085 & 0.176 & 0 & 1.5 \\
\hline 7 & $4-5$ & 0.017 & 0.092 & 0.158 & 12 & 1.5 \\
\hline 8 & $5-6$ & 0.039 & 0.200 & 0.358 & 0 & 1.5 \\
\hline
\end{tabular}

Table B. Load Flow Solutions for the 6-Bus System

\begin{tabular}{|c|c|c|c|c|}
\hline & \multicolumn{2}{|c|}{ Original L. F. } & \multicolumn{2}{c|}{ L. F. After OPF } \\
\hline $\begin{array}{c}\text { Bus } \\
\text { No. }\end{array}$ & $\mathrm{V}$ & $\begin{array}{c}\theta \\
\text { (deg.) }\end{array}$ & $\mathrm{V}$ & $\begin{array}{c}\theta \\
\text { (deg.) }\end{array}$ \\
\hline $1^{*}$ & 1.040 & 0.000 & 1.040 & 0.000 \\
\hline 2 & 1.028 & -5.602 & 1.035 & -1.909 \\
\hline 3 & 1.017 & -7.426 & 1.026 & -3.199 \\
\hline 4 & 1.029 & 1.669 & 1.037 & -2.288 \\
\hline 5 & 1.025 & -5.647 & 1.033 & 0.353 \\
\hline 6 & 1.025 & -4.624 & 1.033 & -0.190 \\
\hline
\end{tabular}

* Bus 1 is the reference bus. Its $P_{G_{1}}+j Q_{G 1}$ changes from $(0.8407-\mathrm{j} 0.0570) \mathrm{pu}$ before OPF to $(0.8138-\mathrm{j} 0.2176) \mathrm{pu}$ after OPF.

\section{BIOGRAPHIES}

Ping Wei (St. M. IEEE) received her M. Eng. degree in electrical engineering from Southeast University, China. She is now a $\mathrm{Ph}$. D. student, Dept. of EEE, the University of Hong Kong. Her research area is power system operation, power market, and operations research applications in power system.

Bin Yuan received his B.S., M.S. and $\mathrm{Ph} . \mathrm{D}$ degrees in Electrical Engineering in 1984, 1987 and 1990 respectively. He joined Tsinghua University, China in 1990. He once worked in Mitsubishi Electric Co. as a visiting researcher and now works in the University of Hong Kong as a visiting scholar. His research area is power system stability, real-time simulation, AI and computer applications in power systems and power market.

Yixin Ni (S. M. IEEE) received her B. Eng., M. Eng., and with the University of Hong Kong. Her research interests are power system stability and control, FACTS, AI tech. applications and power market.

Felix F. Wu (Fellow, IEEE) He received his Ph. D. degree from University of Califomia at Berkeley (UCB). He is now a chair professor of electrical engineering and vice president of the University of Hong Kong. Prior to that he was a professor and vice-chair of Dept. of EECS, UCB. His research interests are electric energy industry restructuring, power system investment planning, design of modern control centers, distribution automation, distributed processing etc. 